\title{
Some new applications of truncated Gauss-Laguerre quadrature formulas
}

\author{
G. Mastroianni • G. Monegato
}

Received: 30 March 2009 / Accepted: 31 March 2009 /

Published online: 24 April 2009

(C) Springer Science + Business Media, LLC 2009

\section{Erratum to: Numerical Algorithms (2008) 49:283-297 DOI 10.1007/s11075-008-9191-x}

In the statement of Lemma 3.1 and in its proof, replace $\frac{3}{2}$ by $\frac{1}{2}$.

The last line of the statement of Theorem 3.2 should be: where $\mathcal{C} \neq \mathcal{C}(m, f)$ and $r$ is an arbitrary positive integer.

The proof of Theorem 3.2 ends at p. 296, before Example 3.3.

In the second line after formula (25), replace $x^{\frac{1}{4}}$ by $x^{\frac{1}{3}}$.

In the headline of Table 11 delete the weight $u$.

The online version of the original article can be found at http://dx.doi.org/10.1007/s11075-008-9191-x.

G. Mastroianni

Dipartimento di Matematica, Università della Basilicata, Potenza, Italy

e-mail: mastroianni@unibas.it

G. Monegato $(\bowtie)$

Dipartimento di Matematica, Politecnico di Torino, Turin, Italy

e-mail: giovanni.monegato@polito.it 